\title{
Comparable outcomes for $\beta$-lactam/ $\beta$-lactamase inhibitor combinations and carbapenems in definitive treatment of bloodstream infections caused by cefotaxime-resistant Escherichia coli or Klebsiella pneumoniae
}

Patrick N A Harris ${ }^{1,2,3^{*}}$, Mo Yin ${ }^{2,3}$, Roland Jureen ${ }^{3,4}$, Jonathan Chew ${ }^{5}$, Jaminah Ali ${ }^{2}$, Stuart Paynter ${ }^{6}$,

David L Paterson ${ }^{1}$ and Paul A Tambyah ${ }^{2,3}$

\begin{abstract}
Background: Extended-spectrum $\beta$-lactamase (ESBL) producing Enterobacteriaceae are often susceptible in vitro to $\beta$-lactam/ $\beta$-lactamase inhibitor (BLBLI) combination antibiotics, but their use has been limited by concerns of clinical inefficacy. We aimed to compare outcomes between patients treated with BLBLIs and carbapenems for bloodstream infection (BSI) caused by cefotaxime non-susceptible (likely ESBL- or AmpC $\beta$-lactamase-producing) Escherichia coli and Klebsiella pneumoniae.

Methods: All adult patients with a BSI caused by cefotaxime non-susceptible E. coli or K. pneumoniae were included from May 2012-May 2013. We compared outcomes between patients who had definitive monotherapy with a carbapenem to those who had definitive monotherapy with a BLBLI.

Results: There were 92 BSIs that fulfilled the microbiological inclusion criteria. 79 (85.9\%) were caused by E. coli and $13(14.1 \%)$ by K. pneumoniae. Four out of $23(17.4 \%)$ patients treated with carbapenem monotherapy and 2 out of 24 (8.3\%) patients treated with BLBLI monotherapy died (adjusted HR for survival 0.91, 95\% Cl 0.13 to 6.28; $\mathrm{p}=0.92$ ). The time to resolution of systemic inflammatory response syndrome (SIRS) criteria did not vary between the treatment groups (adjusted HR 0.91, 95\% Cl 0.32 to 2.59; $\mathrm{p}=0.97$ ). The length of hospital admission post-positive blood culture was slightly longer in patients treated with BLBLIs (median duration 15 vs. 11 days), although this was not significant (adjusted HR 0.62; $95 \% \mathrm{Cl} 0.27$ to 1.42; $\mathrm{p}=0.26$ ). There were no significant differences in subsequent isolation of carbapenem resistant organisms (4.3\% vs. 4.2\%, $p=1.0)$, C. difficile infection (13.0\% vs. $8.3 \%, p=0.67)$ or relapsed $\mathrm{BSI}(0 \%$ vs. $2 \%, \mathrm{p}=0.23)$.
\end{abstract}

Conclusions: BLBLIs appear to have a similar efficacy to carbapenems in the treatment of cefotaxime-resistant E. coli and K. pneumoniae bloodstream infections. Directed therapy with a BLBLI, when susceptibility is proven, may represent an appropriate carbapenem-sparing option.

Keywords: Extended-spectrum $\beta$-lactamase, Enterobacteriaceae, Piperacillin-tazobactam, Carbapenem

\footnotetext{
*Correspondence: p.harris@uq.edu.au

'University of Queensland Centre for Clinical Research, Brisbane, Queensland,

Australia

${ }^{2}$ Department of Infectious Diseases, National University Hospital, Singapore,

Singapore

Full list of author information is available at the end of the article
}

C Biomed Central (c) 2015 Harris et al.; licensee BioMed Central. This is an Open Access article distributed under the terms of the Creative Commons Attribution License (http://creativecommons.org/licenses/by/4.0), which permits unrestricted use, distribution, and reproduction in any medium, provided the original work is properly credited. The Creative Commons Public Domain Dedication waiver (http://creativecommons.org/publicdomain/zero/1.0/) applies to the data made available in this article, unless otherwise stated. 


\section{Introduction}

Gram-negative bacteria that possess extended-spectrum $\beta$-lactamase (ESBL) enzymes have emerged as a major global public health concern in recent years [1]. When first recognized, these resistant isolates were usually implicated in nosocomially-acquired infections or outbreaks [2]. Today ESBL-producers are commonplace in the community $[3,4]$ or in the broader healthcare context, such as residential aged-care facilities $[5,6]$. The burden of disease is particularly marked in the Asian region $[7,8]$. In Singapore, approximately $20 \%$ of $E$. coli and $32 \%$ of Klebsiella spp. are non-susceptible to third-generation cephalosporins [9].

Carbapenems have been regarded as the treatment of choice for serious infections caused by ESBL-producers $[10,11]$. However, the increasing worldwide incidence of ESBL-related infections is driving increased use of carbapenems, leading to selection pressure for carbapenem resistance $[12,13]$. Even brief exposure to a carbapenem can increase the risk of colonisation or infection with a carbapenem-resistant organism [14]. We now face the challenge of emerging carbapenem resistance, largely mediated by the efficient spread of carbapenemases in key Gram-negative pathogens $[15,16]$. Genes coding for carbapenemases are usually co-located with multiple acquired resistance determinants, leaving few effective, low toxicity or non-parenteral options for therapy [17].

In the face of this rapidly changing epidemiology, there is a pressing need to reduce carbapenem overuse. One strategy could be re-evaluating existing agents which have previously been considered ineffective or lack clinical data to support their use. The $\beta$-lactam/ $\beta$-lactamase inhibitor (BLBLI) combination antibiotics, such as amoxicillin-clavu lanate, ticarcillin-clavulanate and piperacillin-tazobactam, have a controversial status in the treatment of infections caused by ESBL-producers [1]. By definition, Ambler class A ESBL enzymes are inhibited by clavulanate or tazobactam in vitro and ESBL producers, especially E. coli, are frequently susceptible to BLBLIs. Yet, there have been concerns that such in vitro susceptibility may not reliably translate into clinical efficacy [18]. This has been based largely on concerns over inoculum effects, the co-location of other beta-lactamase enzymes (which may not be well inhibited by $\beta$-lactamase inhibitors) on acquired plasmids and the potential for additional resistance mechanisms such as alterations in outer membrane proteins [19].

Recent studies suggest that in certain situations, BLBLIs are non-inferior to carbapenems if isolates are susceptible in vitro, [20] especially if the minimum inhibitory concentration (MIC) is low [21]. Such a strategy may represent a reasonable carbapenem-sparing option for treating infections caused by ESBL-producers [19]. This study aimed to examine current treatment strategies for bacteraemia caused by cefotaxime non-susceptible Escherichia coli or Klebsiella spp. in an institution with a relatively high incidence of ESBL-producing isolates. Increasingly, BLBLIs are being considered for treatment of these infections as part of antimicrobial stewardship and carbapenem restriction. As such, we aimed to compare clinical and microbiological outcomes between patients receiving definitive carbapenem therapy with those given piperacillin-tazobactam, as a carbapenemsparing treatment option.

\section{Materials and Methods}

The study has been presented in accordance with the STROBE guidelines on the reporting of observational studies [22].

\section{Objectives}

The aim of the study was to compare the efficacy of BLBLI antibiotics to carbapenems for the treatment of bloodstream infections caused by cefotaxime-resistant (likely ESBL- or AmpC-producing) E. coli or K. pneumoniae. The hypothesis was that there are no significant differences in outcomes for patients treated with a BLBLI or a carbapenem for bloodstream infections caused by cefotaximeresistant E. coli or K. pneumoniae, if these isolates remain susceptible to piperacillin-tazobactam and meropenem in vitro.

\section{Design}

A retrospective observational study examining a cohort of patients with bloodstream infection to compare outcomes following different antibiotic treatments.

\section{Setting}

Patients were identified from admissions to National University Hospital (NUH), a tertiary referral hospital in Singapore with approximately 1,068 beds. The hospital also treats many patients from elsewhere in Asia and the Middle-East. The prevalence of ESBL-producing isolates is relatively high in patients presenting both from the community and with hospital-acquired infection.

\section{Ethics}

The Domain Specific Review Board (DSRB) of the National Healthcare Group (NHG) in Singapore provided ethics approval for this study (NHG DSRB Ref: 2013/00877).

\section{Inclusion and exclusion criteria}

Any adult patient ( $\geq 21$ years) with a bloodstream infection due to E. coli or Klebsiella spp. identified through the NUH microbiology laboratory, defined by at least one monomicrobial positive blood culture between May 2012 to May 2013, was eligible for inclusion. Bacterial isolates were confirmed as cefotaxime non-susceptible, but piperacillin-tazobactam and meropenem susceptible 
by EUCAST standards [23]. Patients were excluded if they showed polymicrobial bacteraemia or if no antimicrobial therapy was given.

\section{Data collection}

Data were obtained by clinical chart review and interrogation of the electronic prescribing and laboratory information systems. A standard clinical record form was used. Demographic information such as age, date of hospital admission and ethnicity were recorded. Community, hospital and healthcare acquisition of bloodstream infection was defined according to standard criteria (defined below) [24]. Concurrent co-morbidities defined at admission were recorded (see Additional file 1). The presence of a medical device in the 7 days prior to bloodstream infection was recorded; this included vascular catheters (peripherally inserted central catheter [PICC], dialysis catheter, central venous catheter or implanted line), central nervous system devices (e.g. external ventricular drain) and urinary catheters or nephrostomy tubes. Relevant therapy in the 30 days prior to bloodstream infection was documented, including cytotoxic chemotherapy, systemic corticosteroids (>15 mg of prednisolone daily or equivalent), anti-TNF (tumour necrosis factor) therapy in last 12 months, other monoclonal antibody therapy, use of immune suppressive agents or radiation therapy. Any surgery in 14 days prior to first blood culture was also recorded. All enrolled patients were stratified using the Charlson co-morbidity index (CCI) using pre-specified definitions (see supplementary material) [25]. Patients admitted to the intensive care unit (ICU) were also assessed using APACHE II scores [26]. Pitt bacteraemia scores were calculated based on clinical parameters measured within 24 hours of initial blood culture collection [27].

The probable source of bacteraemia was assessed according to the available clinical and microbiological information and classified by the investigators using the following categories: urinary tract, central nervous system, pneumonia, intra-abdominal, hepato-biliary, mucositis, line related, musculoskeletal, skin and soft tissue (including burns), surgical site infection, neutropenic sepsis, other or unknown source.

\section{Acquisition status}

Acquisition status was defined as follows, in accordance with standard criteria [24]:

1. Hospital-acquired infection (HAI): afebrile on admission, blood culture positive $>48 \mathrm{~h}$ after admission or within $48 \mathrm{~h}$ after discharge.

2. Community onset: blood culture collected $<48 \mathrm{~h}$ after admission, not admitted or $>48 \mathrm{~h}$ after discharge; then defined as healthcare associated if any of the following: a. Hospitalisation (excluding natural birth) for 2 or more days within 90 days or attendance at emergency department within 2-30 days before bloodstream infection

b. Admission to an out-patient intravenous therapy or hospital-in-the-home service within 2-30 days before bloodstream infection

c. Chronic renal dialysis

d. Residence in long-term care facility

3. Community associated: none of the above

\section{Antibiotic use}

Therapy was defined as empirical if the first dose was given within the first 72 hours following blood culture collection, before results of blood cultures were available (including susceptibility testing); definitive therapy was defined if initiated $>72 \mathrm{~h}$ following initial blood culture collection and for $\geq 50 \%$ of the total treatment duration. All Gramnegative active agents administered were recorded, including doses, frequency and duration in days. Prescribing data were collected using the hospital electronic prescribing system. Adequate empirical therapy was defined as the receipt of at least one parenteral agent (or oral agent with good bioavailability, such as a fluoroquinolone) to which the isolate was subsequently found to be susceptible in vitro. Intravenous doses of piperacillin-tazobactam $4.5 \mathrm{~g}$ 6-hourly or 8-hourly, amoxicillin-clavulanate $1.2 \mathrm{~g}$ 8-hourly, meropenem $1 \mathrm{~g}$ 8-hourly, ertapenem $1 \mathrm{~g}$ 24-hourly or imipenem $500 \mathrm{mg}$ 6-hourly were used (with adjustment for renal dysfunction) as per local prescribing guidelines.

\section{Vital sign measurement}

Maximum and minimum temperatures $\left(\mathrm{T}_{\max }\right.$ and $\left.\mathrm{T}_{\min }\right)$, maximal heart rate, maximal respiratory rate, highest total white cell count (if tested), lowest systolic blood pressure, use of a vasopressor agent and results of any repeat blood cultures (if collected) were recorded daily for seven days.

\section{Outcome measures}

1. Days to resolution of systemic inflammatory response syndrome (SIRS). SIRS was defined as being present if $\geq 2$ of the following were recorded:

a. $\mathrm{T}_{\max }>38^{\circ} \mathrm{C}$ or $\mathrm{T}_{\min }<36^{\circ} \mathrm{C}$

b. Lowest systolic blood pressure $<90 \mathrm{mmHg}$ and/or inotrope requirement,

c. Respiratory rate $>20 / \mathrm{min}$ or arterial $\mathrm{PaCO}_{2}<32 \mathrm{~mm}$ $\mathrm{Hg}$ if ventilated,

d. Maximal heart rate $>90 / \mathrm{min}$,

e. Total white cell count $>12 \times 10^{9} / \mathrm{L}$ or $<4 \times 10^{9} / \mathrm{L}$

Days to resolution of SIRS was calculated from the date of initial blood culture collection to the first 
Table 1 Susceptibility profiles of $E$. coli or K. pneumoniae isolated from blood cultures with resistance to ceftriaxone during study period

\begin{tabular}{|c|c|c|c|c|c|c|c|c|c|c|c|c|c|c|c|c|c|}
\hline \multicolumn{8}{|c|}{ Susceptibility category } & \multicolumn{10}{|c|}{ Antimicrobial agent tested } \\
\hline E. coli & AMP & AMC & TZP & CXM & CRO & CTX & $\mathrm{CAZ}$ & FEP & FOX & IPM & MEM & ETP & LVX & CIP & SXT & GEN & AMK \\
\hline R (\%) & 100 & 60 & 0 & 100 & 100 & 100 & 96 & 0 & 11 & 0 & 0 & 0 & 71 & 71 & 59 & 35 & 0 \\
\hline I (\%) & 0 & 0 & 0 & 0 & 0 & 0 & 4 & 4 & 20 & 0 & 0 & 0 & 0 & 3 & 0 & 0 & 3 \\
\hline S (\%) & 0 & 40 & 100 & 0 & 0 & 0 & 0 & 96 & 68 & 100 & 100 & 100 & 29 & 27 & 41 & 65 & 97 \\
\hline Total tested & 79 & 73 & 79 & 79 & 79 & 79 & 79 & 79 & 79 & 79 & 79 & 79 & 79 & 79 & 79 & 79 & 79 \\
\hline \multicolumn{18}{|c|}{ K. pneumoniae } \\
\hline $\mathrm{R}(\%)$ & 100 & 45 & 0 & 100 & 100 & 100 & 100 & 92 & 0 & 0 & 0 & 0 & 15 & 30 & 85 & 31 & 0 \\
\hline I (\%) & 0 & 0 & 0 & 0 & 0 & 0 & 0 & 8 & 8 & 0 & 0 & 0 & 0 & 40 & 0 & 0 & 0 \\
\hline S (\%) & 0 & 55 & 100 & 0 & 0 & 0 & 0 & 0 & 92 & 100 & 100 & 100 & 85 & 30 & 15 & 69 & 100 \\
\hline Total tested & 13 & 11 & 13 & 13 & 13 & 13 & 13 & 13 & 13 & 13 & 13 & 13 & 13 & 10 & 13 & 13 & 13 \\
\hline
\end{tabular}

$\mathrm{R}=$ resistant $\mathrm{I}=$ intermediate $\mathrm{S}=$ susceptible; $\mathrm{AMP}=$ ampicillin, $\mathrm{AMC}=$ amoxicillin-clavulanate, $\mathrm{TZP}=$ piperacillin-tazobactam, $\mathrm{CXM}=$ cefuroxime, $\mathrm{CRO}=\mathrm{ceftriaxone}$, $\mathrm{CTX}=$ cefotaxime, $\mathrm{CAZ}=$ ceftazidime, $\mathrm{FEP}=$ cefepime, $\mathrm{FOX}=$ cefoxitin, IMP = imipenem, MEM = meropenem, $\mathrm{ETP}=$ ertapenem, $\mathrm{LVX}=$ levofloxacin, $\mathrm{CIP}=\mathrm{ciprofloxacin}$,

$\mathrm{SXT}=$ trimethoprim-sulphamethoxazole, $\mathrm{GEN}=$ gentamicin, $\mathrm{AMK}=$ amikacin.

day where the patients did not fulfil SIRS criteria (as defined above). Patients were assumed to have resolution of SIRS on the date of discharge (if they did not have resolution of SIRS previously using the above criteria). Patients who died were included in the analysis, and defined as never having resolution of SIRS. Where daily white cell count values were missing, the value was imputed by calculating the midpoint difference between the adjacent day values. Patients for whom SIRS criteria could not be ascertained due to missing data were excluded from this part of the analysis.

2. All-cause mortality at 30 days post initial positive blood culture.

3. Identification of a carbapenem or piperacillintazobactam resistant isolate in subsequent 30 days or identification of Clostridium difficile infection.

4. Microbiological relapse (positive blood culture $>72 \mathrm{~h}$ after initiation of definitive therapy and up to 30 days) with same organism as original initial blood culture.

5. Length of hospital stay post first positive blood culture. Patients who died were excluded from this analysis.

\section{Microbiological testing}

Blood cultures were inoculated into BacT/Alert bottles (BioMerieux; Marcy-L'Étiole, France) and incubated for up to 5 days. Positive cultures were sub-cultured and identified to species level by standard laboratory methods, including MALDI-TOF (Bruker Daltoniks GmHB; Bremen, Germany) and Vitek2 (BioMerieux). Susceptibility testing was performed using Vitek2 microbroth dilution according to EUCAST interpretative standards [23]. Phenotypic testing or molecular methods were not routinely used to confirm ESBL or plasmid-mediated AmpC beta-lactamase production. Antibiotic reporting was at the discretion of the duty microbiologist at the time of the bloodstream infection. All positive blood cultures reported within the 30-day follow-up period were also recorded.

\section{Statistical analysis}

Categorical data were presented as proportions and scale data using median and interquartile ranges. Patients who received carbapenems or BLBLIs as definitive monotherapy were identified and compared. Groups given other non-carbapenem or non-BLBLI therapy and combination therapy were excluded. Survival curves for mortality, days to resolution of SIRS, and length of hospital stay were presented using Kaplan-Meier curves. Cox proportional hazards regression was used to assess potential confounders identified a priori (age, acquisition status, Pitt bacteraemia score, ICU admission, organism, and whether or not the patient had received appropriate empirical therapy). Potential confounders were included in the final regression model if they caused a change in the main effect (hazard ratio according to definitive treatment) by $5 \%$ or more when included in bivariate analysis with definitive treatment. Statistical analysis was performed using Stata (StataCorp; Texas, USA).

Table 2 MIC distributions for cefotaxime-resistant and piperacillin-tazobactam susceptible $E$. coli and $K$. pneumoniae from blood culture isolates tested by Vitek2

\begin{tabular}{llrlrl}
\hline AMC MIC & $\mathbf{N}(\%)$ & FOX MIC & $\mathbf{N}(\%)$ & TZP MIC & $\mathbf{N}(\%)$ \\
\hline 4 & $19(21.8)$ & $<=4$ & $60(72.3)$ & $<=4$ & $65(70.7)$ \\
8 & $19.0(21.8)$ & 8 & $6(7.2)$ & 8.0 & $27.0(29.3)$ \\
16 & $32(36.8)$ & 16 & $8(9.6)$ & - & - \\
$>=32$ & $17(19.5)$ & 32 & $9(10.8)$ & - & - \\
- & - & $>=64$ & $9(10.8)$ & - & - \\
Total & 87 & & 83 & & 92 \\
\hline
\end{tabular}

AMC = amoxicillin-clavulanate, FOX = cefoxitin, TZP = piperacillin-tazobactam. 


\section{Results}

During the period from May 2012 to May 2013 there were there were $476 \mathrm{E}$. coli and $328 \mathrm{~K}$. pneumoniae bloodstream infections in patients $>=21$ years old. Out of the total 804 bloodstream infection events, there were 92 (11.4\%) that fulfilled the microbiological inclusion criteria, of which 79 (85.9\%) were caused by E. coli and 13 (14.1\%) by $K$. pneumoniae. Susceptibility patterns are summarized in Table 1 and MIC distributions for amoxicillinclavulanate, piperacillin-tazobactam and cefoxitin are shown in Table 2. One patient was excluded as they were discharged without any antibiotic treatment. The median age was 75 years (range $23-100$ years; IQR 21 ) and 53.8\% were female. Of the 91 patients given treatment, 44 received definitive treatment that did not include a BLBLI or a carbapenem or were given combination definitive therapy, leaving 47 patients eligible for inclusion in the analysis for definitive therapy (24 receiving a BLBLI and 23 a carbapenem) (see Figure 1). Definitive carbapenem monotherapy included $60.9 \%$ given meropenem, $34.8 \%$ imipenem and $4.3 \%$ ertapenem; for definitive monotherapy with a BLBLI, 95.8\% were given piperacillin-tazo bactam and $4.2 \%$ amoxicillin-clavulanate. In the carbapenem treated group, $17.4 \%$ received 'step-down' once daily therapy with ertapenem following initial therapy with imipenem or meropenem. In the BLBLI treated group, 8.3\% were given 'step-down' therapy with amoxicillinclavualante following piperacillin-tazobactam. Other antibiotic choices, along with the baseline characteristics are reported in Table 3. Mortality was relatively infrequent: four out of $23(17.4 \%)$ patients treated with carbapenem monotherapy and 2 out of $24(8.3 \%)$ patients treated with BLBLI monotherapy (Figure 2). On univariate analysis of mortality, a non-significant result favouring BLBLIs was seen, but this was diminished after adjustment for confounders (see Table 4). The HR for survival at 30 days was 0.91 (95\% CI 0.13 to $6.28 ; \mathrm{p}=0.92$ ) after adjustment for ICU admission, infecting organism, and Pitt score. There was no difference in the time to resolution of SIRS between the two definitive treatment groups (HR 0.91, 95\% CI 0.32 to 2.59; $\mathrm{p}=0.97$ ) (Figure 3 and Table 4). For the analysis of the length of hospital stay post positive blood culture, four patients were excluded because they had prolonged hospital admission ( $>40$ days) due to unrelated factors. The length of hospital stay post positive blood culture was slightly longer in patients treated with BLBLI compared to carbapenem. For those treated with a BLBLI, the median length of stay was 15 days [IQR 10 to 19 days] while for those given a carbapenem, the median length of stay was 11 days [IQR 8 to 20 days]. In the Cox regression analysis, this difference was not statistically significant (HR 0.62, 95\% CI 0.27 to 1.42; $\mathrm{p}=0.26$ ) (Figure 4 and Table 4). There were no significant differences in subsequent isolation of a carbapenem resistant organism
Table 3 Baseline characteristics of patients given definitive monotherapy with a BLBLI or carbapenem

\begin{tabular}{|c|c|c|c|}
\hline \multirow[t]{2}{*}{$\begin{array}{l}\text { Patient } \\
\text { characteristic }\end{array}$} & \multicolumn{2}{|c|}{$\begin{array}{l}\text { Definitive } \\
\text { treatment cohort }\end{array}$} & \multirow{2}{*}{$\begin{array}{l}\text { Total study } \\
\text { population } \\
\mathrm{N}=91\end{array}$} \\
\hline & $\begin{array}{l}\text { BLBLI } \\
(\mathrm{N}=24)\end{array}$ & $\begin{array}{l}\text { Carbapenem } \\
(\mathrm{N}=23)\end{array}$ & \\
\hline $\begin{array}{l}\text { Age, median [IQR], } \\
\text { years }\end{array}$ & 77 [61-83] & 77 [68-83] & 75 [62-83] \\
\hline Female & $13(54 \%)$ & $12(52 \%)$ & 49 (54\%) \\
\hline Hospital acquired & $7(29 \%)$ & $4(17 \%)$ & $20(22 \%)$ \\
\hline Community acquired & 7 (29\%) & $9(39 \%)$ & $42(46 \%)$ \\
\hline Healthcare associated & $10(42 \%)$ & $10(43 \%)$ & $29(32 \%)$ \\
\hline $\mathrm{CCl}$, median [IQR] & $2[1-4]$ & $2[1-5]$ & $2[1-4]$ \\
\hline Pitt score, median [IQR] & $1[0-2]$ & $1[0-3]$ & $1[0-2]$ \\
\hline $\begin{array}{l}\text { APACHEII (if ICU), } \\
\text { median [IQR] }\end{array}$ & 26 & 20 & 24 [15-28] \\
\hline ICU admission & $2(8 \%)$ & $5(22 \%)$ & $11(12.1)$ \\
\hline E. coli & $22(92 \%)$ & 17 (74\%) & $79(87 \%)$ \\
\hline
\end{tabular}

Source:

Hepato-biliary

Urinary tract

Neutropenic sepsis

Other/unknown source

$2(8 \%)$

Co-morbidity/devices:

Moderate to severe

liver disease

Diabetes without end organ damage

Diabetes with organ damage

Moderate to severe renal disease

Metastatic solid tumour

Leukaemia or lymphoma

Urinary device

$9(38 \%) \quad 13(57 \%)$

$12(13 \%)$

(4\%) $\quad 0(0 \%)$

$43(47 \%)$

$12(50 \%) \quad 8(34 \%)$

$4(4 \%)$

$32(35 \%)$

Immunosuppressive treatments

$3(13 \%)$

$1(4 \%)$

$7(8 \%)$

$6(25 \%) \quad 5(22 \%)$

$21(23 \%)$

Empirical therapy:

3GC

BLBLI

Carbapenem

Other*

$4(17 \%) \quad 5(22 \%) \quad 15(17 \%)$

$4(17 \%) \quad 7(31 \%)$

19 (21\%)

$1(4 \%)$

$1(4 \%)$

$4(4 \%)$

$1(4 \%) \quad 2(9 \%)$

$7(8 \%)$

$15(17 \%)$

$12(13 \%)$

$2(8 \%) \quad 4(17 \%)$

$(13 \%)$

Appropriate empirical therapy

*Including combinations of carbapenem/BLBL/3CG.

$\mathrm{CCl}=$ Charlson Co-morbidity index, IQR = Inter-quartile range, $\mathrm{ICU}=$ intensive care unit, $3 \mathrm{GC}=$ third-generation cephalosporins,

$\mathrm{BLBLI}=$ beta-lactam/beta-lactamase inhibitor.

(4.3\% vs. $4.2 \%, \mathrm{p}=1.0)$, C. difficile infection $(13.0 \%$ vs. $8.3 \%, \mathrm{p}=0.67)$ or relapsed bloodstream infection $(0 \% \mathrm{vs}$. $2 \%, \mathrm{p}=0.23)$. 
Bloodstream infection with E. coli or K. pneumoniae resistant to cefotaxime, susceptible to piperacillin-tazobactam $(n=92)$

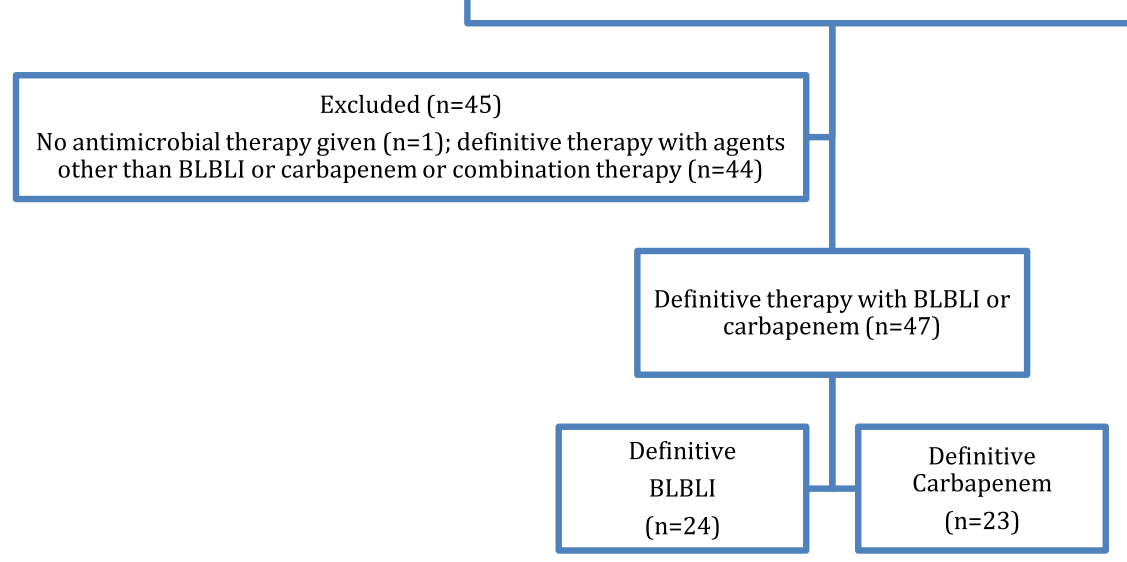

Figure 1 Patient inclusion flowchart - treatment with BLBLI or carbapenem.

\section{Discussion}

In this retrospective observational study, use of a BLBLI as definitive monotherapy for the treatment of cefotaxime non-susceptible $E$. coli or $K$. pneumoniae bloodstream infection was not associated with worse outcome when compared to the use of a carbapenem - often considered standard therapy for such infections [10]. Not only were there no significant differences in 30-day mortality, but there were also no differences in resolution of SIRS criteria over 7 days post bacteraemia. Although event rates were low, there were also no differences in rates of relapsed bacteraemia, $C$. difficile infection or subsequent isolation of a multi-resistant organism. There was a trend towards a longer length of hospital stay in the BLBLItreated group; although this was not significant (the Cox regression analysis of the length of hospital stay had a power of $55 \%$ to detect a hazard ratio of 0.5 ).

These findings are broadly similar to a prospective study addressing this question from Rodriguez-Bano et al. [20] and a meta-analysis that examined studies that reported outcomes for patients treated with BLBLIs for bacteraemia caused by ESBL-producers [28]. In both

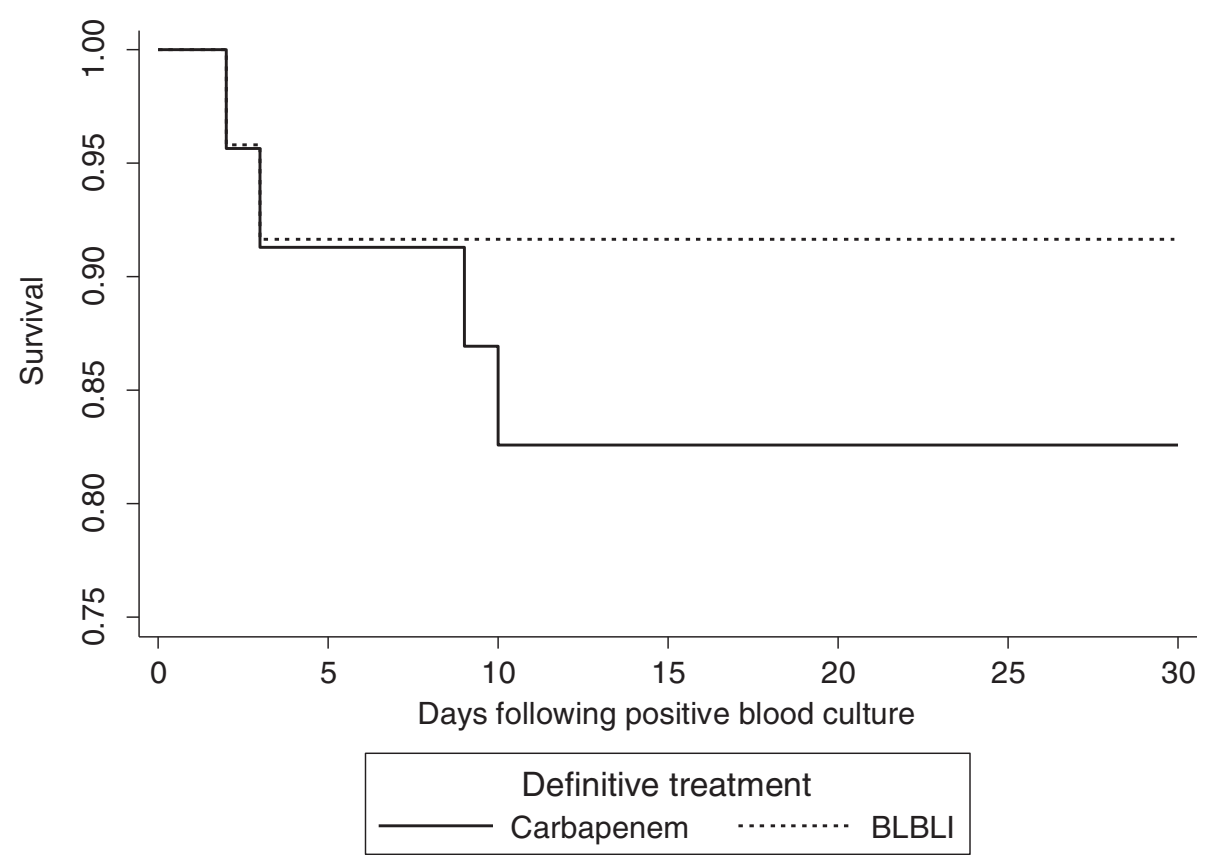

Figure 2 30-day mortality for patients treated with BLBLI or carbapenem as definitive monotherapy. 
Table 4 Results of Cox regression analyses

\begin{tabular}{|c|c|c|c|c|}
\hline Outcome & Definitive treatment & $\mathbf{n}$ & Crude hazard ratio $(95 \% \mathrm{Cl})$ & Adjusted hazard ratio $(95 \% \mathrm{Cl})$ \\
\hline \multirow[t]{2}{*}{30 day mortality } & Carbapenem & 20 & 1 & 1 \\
\hline & BLBLI & 21 & 0.47 (0.09 to 2.59$)$ & $0.91(0.13 \text { to } 6.28)^{*}$ \\
\hline \multirow[t]{2}{*}{ Resolution of SIRS } & Carbapenem & 14 & 1 & 1 \\
\hline & BLBLI & 14 & 1.19 (0.44 to 3.19$)$ & $0.91(0.32 \text { to } 2.59)^{*}$ \\
\hline \multirow[t]{2}{*}{ Hospital discharge } & Carbapenem & 16 & 1 & 1 \\
\hline & BLBLI & 16 & 0.74 (0.38 to 1.41$)$ & $0.62(0.27 \text { to } 1.42)^{*}$ \\
\hline
\end{tabular}

*Adjusted for ICU admission, infecting organism, Pitt score.

studies, there were no differences in mortality between patients given BLBLIs when compared with carbapenems for empirical or definitive therapy. As such, these studies would support the concept that BLBLIs, at least, represent a safe carbapenem-sparing option when susceptibility is proven, despite the likely presence of a broad-spectrum beta-lactamase.

Limitations of the study are acknowledged. The total patient cohort was relatively small, especially in the definitive treatment cohort receiving monotherapy with a BLBLI or carbapenem. A significant proportion of patients received either sequential monotherapy with different agents, or various combination therapies, and so were excluded from the analysis thus reducing the sample size. As such, the study was underpowered to detect true differences in infrequent outcomes, particularly for mortality at 30 days. Given the retrospective nature of the study, potential confounders are likely. These might include the propensity to receive a carbapenem (which may occur in patients with a higher risk of mortality) potentially over-estimating the relative efficacy of BLBLIs. We have adjusted for several potential confounding factors where appropriate, however residual confounding from these factors, or confounding from other unmeasured factors, may remain. Although Pitt scores are predictive for mortality in bloodstream infection, additional measures (such as the presence of septic shock or other markers of illness severity at presentation) may have provided further information on potential confounders, but were not available.

Resistance to third-generation cephalosporins, using current EUCAST criteria, is a sensitive but not highly specific marker for ESBL-production. Plasmid-mediated AmpC beta-lactamase acquisition has become widespread in recent years in these species and may provide a similar resistance profile, although are usually also resistant to cefoxitin (in contrast to ESBL-producers). This is of relevance because $A m p C$ enzymes are less effectively inhibited by tazobactam, which could limit clinical efficacy. In this study we were not able to confirm the beta-lactamase types in blood culture isolates. However, amongst E. coli and $K$. pneumoniae, $31 \%$ and $8 \%$ respectively tested nonsusceptible (resistant or intermediate) to cefoxitin, suggesting that plasmid-AmpC may occur frequently within E. coli in this population. However, ESBLs may themselves cause elevated MICs to cefoxitin, so extrapolation from

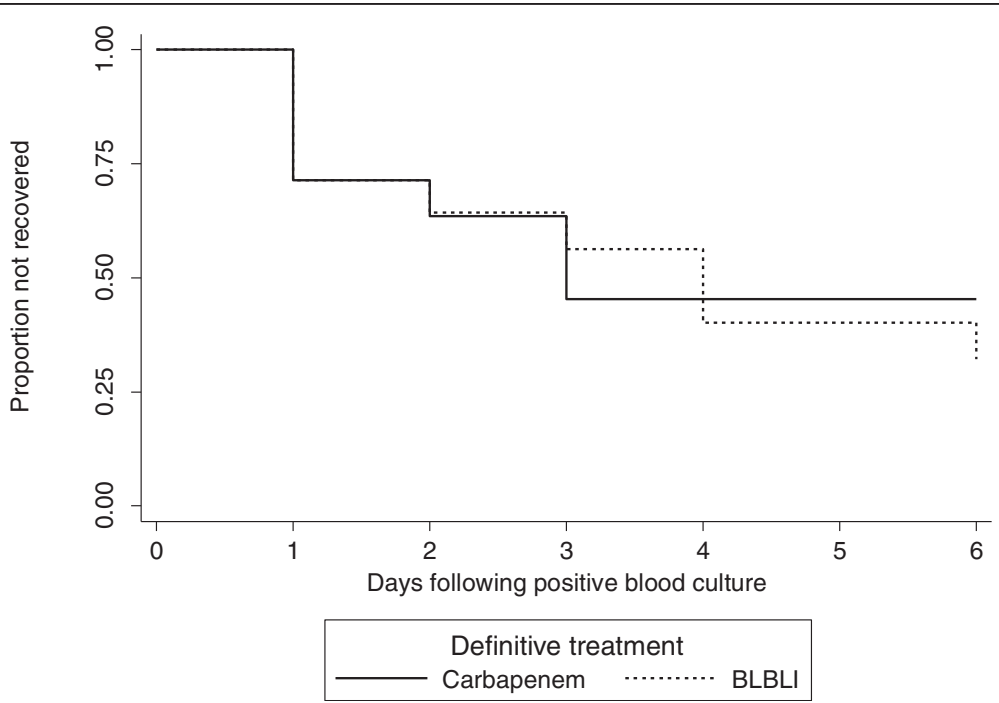

Figure 3 Days to recovery from SIRS ( $<2$ SIRS criteria or discharge). 


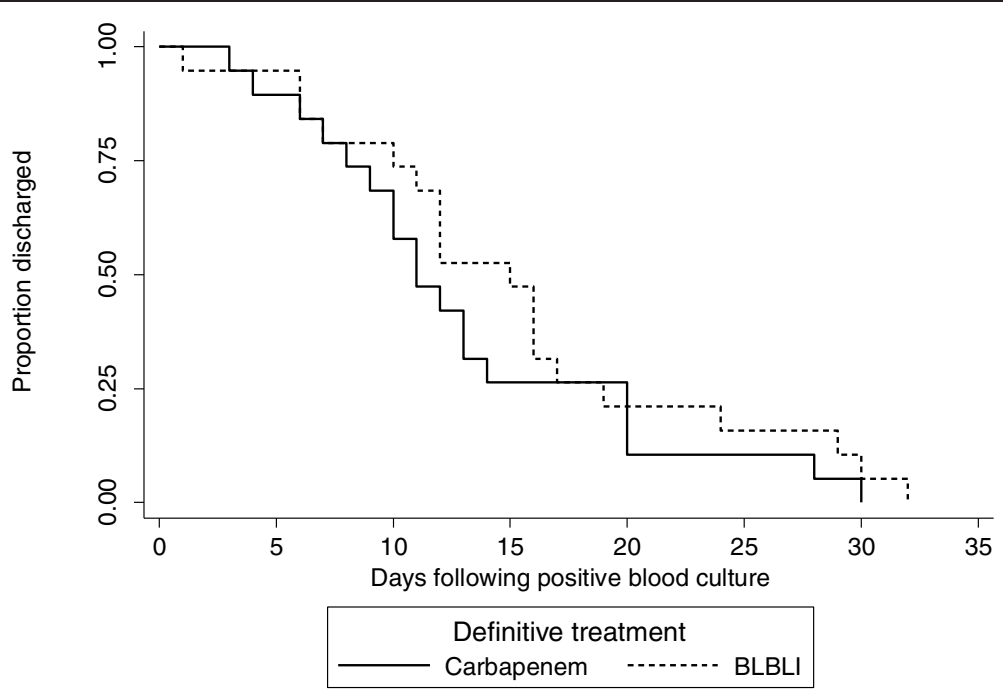

Figure 4 Length of hospital admission post-positive blood culture for patients treated with BLBLI or carbapenem as definitive monotherapy.

the antibiogram alone can be misleading. A cefoxitin MIC $\geq 32 \mu \mathrm{g} / \mathrm{mL}$ has been suggested as a useful screening marker for selecting isolates for confirmatory tests of AmpC production [29]. It is also acknowledged that MIC determination with the Vitek2 instrument, as used in this study, is not a reference method. In common with several other countries, the predominant ESBLs found in Enterobacteriaceae in Singapore are CTX-M types, but plasmidmediated AmpC-producers are increasingly seen [30,31]. In an ongoing study using whole genome sequencing to characterise third-generation cephalosporin-resistant but piperacillin-tazobactam susceptible E. coli or K. pneumoniae from BSIs in Singapore, CTX-M-type ESBLs predominated (found in more than $85 \%$ of isolates) although plasmid-mediated AmpC (CMY- or DHA-like) $\beta$-lactamases were present in around 10\% (in-house unpublished data). There has also been a marked increase in the numbers of isolates with acquired carbapenemases in Singapore, [32] highlighting the need to define alternatives to carbapenems where possible.

Further work is needed to definitively test the concept that BLBLIs are, in general, a safe and effective carbapenem-sparing option for the treatment of bloodstream infections caused by third-generation cephalos porin-resistant E. coli or K. pneumoniae. A large international retrospective observational study has recently reported similar findings to this study [33]. However, studies of this nature are always prone to bias, leaving ongoing uncertainty as to the clinical efficacy of BLBLIs against ESBL-producers. There also remains debate over the effect of MICs that fall in the higher end of the susceptible range, especially for infection outside the urinary tract [34]. A recent retrospective study compared the empirical use of piperacillin-tazobactam with carbapenems for bloodstream infections caused by ESBL-producers, and reported an adjusted risk of death in patients given piperacillin-tazobactam as 1.92 times higher than those given carbapenem therapy (95\% CI, 1.07-3.45) [35]. As such, there exists considerable uncertainty in the role of BLBLIs for the treatment of bloodstream infection caused by ESBL-producers. Ideally, such questions should be answered in a randomised controlled trial. Such a study is now underway across several Australasian sites, including Singapore (the 'MERINO' trial, registered at www.clinicaltrials.gov; NCT02176122) and aims to be completed by 2018 .

\section{Conclusions}

In this retrospective study, comparable outcomes were seen for patients given definitive treatment with BLBLIs or carbapenems for bloodstream infections caused by cefotaxime non-susceptible E. coli or K. pneumoniae in terms of all-cause mortality, resolution of SIRS, length of stay or bacteraemia relapse. There were also no significant differences in subsequent infection or colonisation with a multi-resistant organism or $C$. difficile infection. However, larger studies adequately powered to detect differences in mortality, preferably in the form of multicentre randomised trials, are needed before such a strategy can be recommended as standard care.

\section{Additional file}

Additional file 1: co-morbidity definitions.

\section{Abbreviations}

ESBL: Extended-spectrum $\beta$-lactamase; BLBLI: $\beta$-lactam/ $\beta$-lactamase inhibitor; BSI: Bloodstream infection; SIRS: Systemic inflammatory response syndrome; 
EUCAST: European Committee on Antimicrobial Susceptibility Testing; PICC: Peripherally inserted central catheter; TNF: Tumour necrosis factor; ICU: Intensive care unit; APACHE: Acute Physiology and Chronic Health Evaluation; HAl: Hospital-acquired infection; MALDI-TOF: Matrix Assisted Laser Desorption lonization Time-of-Flight; CCl: Charlson co-morbidity index; MIC: Minimum inhibitory concentration; HR: Hazard ratio.

\section{Competing interests}

PAT has received research support from Baxter, ADAMAS, Merlion Pharmaceuticals, Sanofi Pasteur, Fabentech and Inviragen. He has also received honoraria from Novartis and AstraZeneca. DLP has participated in advisory boards and received honoraria from AstraZeneca, Merck, Pfizer, Bayer, Cubist and Leo Pharmaceuticals. All other authors have no competing interest to declare.

\section{Authors' contributions}

PAT, PH and DLP initiated the concept for the study. PH designed the study, wrote the first and final drafts of the manuscript and analysed the data. MY, $\mathrm{PH}, \mathrm{JC}$ and $\mathrm{PH}$ collected the clinical data. RJ provided the microbiological data. SP provided statistical support. All authors read and approved the final manuscript.

\section{Acknowledgements}

Many thanks to Dr. Anupama Vasudevan who assisted with data management.

\section{Funding}

No external funding was required for the project. PH is supported by an Australian Postgraduate Award with a top-up scholarship from the University of Queensland Centre for Clinical Research.

\section{Author details}

${ }^{1}$ University of Queensland Centre for Clinical Research, Brisbane, Queensland, Australia. ${ }^{2}$ Department of Infectious Diseases, National University Hospital, Singapore, Singapore. ${ }^{3}$ Yong Loo Lin School of Medicine, National University of Singapore, Singapore, Singapore. ${ }^{4}$ Department of Laboratory Medicine, National University Hospital, Singapore, Singapore. ${ }^{5}$ International Medical University, Kuala Lumpur, Malaysia. ${ }^{6}$ School of Population Health, University of Queensland, Brisbane, QLD, Australia.

Received: 7 December 2014 Accepted: 1 April 2015 Published online: 23 April 2015

\section{References}

1. Pitout JD, Laupland KB. Extended-spectrum beta-lactamase-producing Enterobacteriaceae: an emerging public-health concern. Lancet Infect Dis. 2008:8(3):159-66. doi:10.1016/S1473-3099(08)70041-0.

2. Medeiros AA. Nosocomial outbreaks of multiresistant bacteria: extendedspectrum beta-lactamases have arrived in North America. Ann Intern Med. 1993;119(5):428-30.

3. Doi Y, Park YS, Rivera Jl, Adams-Haduch JM, Hingwe A, Sordillo EM, et al. Community-associated extended-spectrum beta-lactamase-producing Escherichia coli infection in the United States. Clin Infect Dis. 2013;56(5):641-8. doi:10.1093/cid/cis942.

4. Ben-Ami R, Rodriguez-Bano J, Arslan H, Pitout JD, Quentin C, Calbo ES, et al. A multinational survey of risk factors for infection with extended-spectrum beta-lactamase-producing enterobacteriaceae in nonhospitalized patients. Clin Infect Dis. 2009:49(5):682-90. doi:10.1086/604713.

5. Stuart RL, Kotsanas D, Webb B, Vandergraaf S, Gillespie EE, Hogg GG, et al. Prevalence of antimicrobial-resistant organisms in residential aged care facilities. Med J Aust. 2011;195(9):530-3.

6. Rooney PJ, O'Leary MC, Loughrey AC, McCalmont M, Smyth B, Donaghy P, et al. Nursing homes as a reservoir of extended-spectrum beta-lactamase (ESBL)-producing ciprofloxacin-resistant Escherichia coli. J Antimicrob Chemother. 2009:64(3):635-41. doi:10.1093/jac/dkp220.

7. Chen YH, Hsueh PR, Badal RE, Hawser SP, Hoban DJ, Bouchillon SK, et al. Antimicrobial susceptibility profiles of aerobic and facultative Gram-negative bacilli isolated from patients with intra-abdominal infections in the Asia-Pacific region according to currently established susceptibility interpretive criteria. J Infect. 2011;62(4):280-91.
8. Molton JS, Tambyah PA, Ang BS, Ling ML, Fisher DA. The Global Spread of Healthcare-Associated Multidrug-Resistant Bacteria: A Perspective From Asia. Clin Infect Dis. 2013;56(9):1310-8.

9. Hsu LY, Tan TY, Tam VH, Kwa A, Fisher DA, Koh TH, et al. Surveillance and correlation of antibiotic prescription and resistance of Gram-negative bacteria in Singaporean hospitals. Antimicrob Agents Chemother. 2010;54(3):1173-8. doi:10.1128/AAC.01076-09.

10. Paterson DL, Bonomo RA. Extended-spectrum beta-lactamases: a clinical update. Clin Microbiol Rev. 2005;18(4):657-86.

11. Nicolau DP. Carbapenems: a potent class of antibiotics. Expert Opin Pharmacother. 2008:9(1):23-37. doi:10.1517/14656566.9.1.23.

12. McLaughlin M, Advincula MR, Malczynski M, Qi C, Bolon M, Scheetz MH. Correlations of antibiotic use and carbapenem resistance in enterobacteriaceae. Antimicrob Agents Chemother. 2013;57(10):5131-3. doi:10.1128/AAC.00607-13.

13. Chang H-J, Hsu P-C, Yang C-C, Kuo A-J, Chia J-H, Wu T-L, et al. Risk factors and outcomes of carbapenem-nonsusceptible Escherichia coli bacteraemia: A matched case-control study. J Microbiol Immunol Infect. 2011;44(2):125-30.

14. Armand-Lefevre L, Angebault C, Barbier F, Hamelet E, Defrance G, Ruppe E, et al. Emergence of imipenem-resistant gram-negative bacilli in intestinal flora of intensive care patients. Antimicrob Agents Chemother. 2013;57(3):1488-95.

15. Nordmann P, Dortet L, Poirel L. Carbapenem resistance in Enterobacteriaceae: here is the storm! Trends Mol Med. 2012;18(5):263-72.

16. Glasner C, Albiger B, Buist G, Tambic Andrasevic A, Canton R, Carmeli Y et al. Carbapenemase-producing Enterobacteriaceae in Europe: a survey among national experts from 39 countries, February 2013. Euro surveill. 2013;18(28). Available at: http://www.eurosurveillance.org/NiewArticle. aspx? Articleld=20525.

17. Akova M, Daikos GL, Tzouvelekis L, Carmeli Y. Interventional strategies and current clinical experience with carbapenemase-producing Gram-negative bacteria. Clin Microbiol Infect. 2012;18(5):439-48.

18. Perez F, Bonomo RA. Can we really use beta-lactam/beta-lactam inhibitor combinations for the treatment of infections caused by extended-spectrum beta-lactamase-producing bacteria? Clin Infect Dis. 2012;54(2):175-7.

19. Harris PN, Tambyah PA, Paterson DL. beta-lactam and beta-lactamase inhibitor combinations in the treatment of extended-spectrum beta-lactamase producing Enterobacteriaceae: time for a reappraisal in the era of few antibiotic options? Lancet Infect Dis. 2015;15(4):475-85.

20. Rodriguez-Bano J, Navarro MD, Retamar P, Picon E, Pascual A. ExtendedSpectrum Beta-Lactamases-Red Espanola de Investigacion en Patologia Infecciosa/Grupo de Estudio de Infeccion Hospitalaria G. beta-Lactam/betalactam inhibitor combinations for the treatment of bacteremia due to extended-spectrum beta-lactamase-producing Escherichia coli: a post hoc analysis of prospective cohorts. Clin Infect Dis. 2012;54(2):167-74.

21. Retamar P, Lopez-Cerero L, Muniain MA, Pascual A, Rodriguez-Bano J, Group E-RG. Impact of the MIC of piperacillin-tazobactam on the outcome of patients with bacteremia due to extended-spectrum-beta-lactamase-producing Escherichia coli. Antimicrob Agents Chemother. 2013;57(7):3402-4. doi:10.1128/AAC.00135-13.

22. von Elm E, Altman DG, Egger M, Pocock SJ, Gotzsche PC, Vandenbroucke $J \mathrm{P}$, et al. The Strengthening the Reporting of Observational Studies in Epidemiology (STROBE) statement: guidelines for reporting observational studies. J Clin Epidemiol. 2008;61(4):344-9. doi:10.1016/j.jclinepi.2007.11.008

23. European Committee on Antimicrobial Susceptibility Testing. Breakpoint tables for interpretation of MICs and zone diameters version 5.0. 2015. www.eucast.org.

24. Friedman ND, Kaye KS, Stout JE, McGarry SA, Trivette SL, Briggs JP, et al. Health care-associated bloodstream infections in adults: a reason to change the accepted definition of community-acquired infections. Ann Intern Med. 2002;137(10):791-7.

25. Charlson M, Szatrowski P, Peterson J, Gold J. Validation of a combined comorbidity index. J Clin Epidemiol. 1994;47(11):1245-51.

26. Knaus WA, Draper EA, Wagner DP, Zimmerman JE. APACHE II: a severity of disease classification system. Crit Care Med. 1985;13(10):818-29.

27. Paterson DL, Ko WC, Von Gottberg A, Mohapatra S, Casellas JM, Goossens H, et al. International prospective study of Klebsiella pneumoniae bacteremia: implications of extended-spectrum beta-lactamase production in nosocomial Infections. Ann Intern Med. 2004;140(1):26-32.

28. Vardakas KZ, Tansarli GS, Rafailidis PI, Falagas ME. Carbapenems versus alternative antibiotics for the treatment of bacteraemia due to 
Enterobacteriaceae producing extended-spectrum beta-lactamases: a systematic review and meta-analysis. J Antimicrob Chemother. 2012;67(12):2793-803.

29. Pitout JD, Le PG, Moore KL, Church DL, Gregson DB. Detection of AmpC beta-lactamases in Escherichia coli, Klebsiella spp., Salmonella spp. and Proteus mirabilis in a regional clinical microbiology laboratory. Clinical microbiology and infection : the official publication of the European Society of Clinical Microbiology and. Infectious Diseases. 2010;16(2):165-70. doi:10.1111/j.1469-0691.2009.02756.x.

30. Sheng W-H, Badal RE, Hsueh P-R. Distribution of extended-spectrum $\beta$ lactamases, AmpC $\beta$-lactamases, and carbapenemases among enterobacteriaceae isolates causing intra-abdominal infections in the Asia-pacific region: results of the study for monitoring antimicrobial resistance trends (SMART). Antimicrob Agents Chemother. 2013;57(7):2981-8. doi:10.1128/AAC.00971-12.

31. Koh TH. Gram-negative resistance in Singapore: a historical perspective. Ann Acad Med Singap. 2008;37(10):847-54.

32. Koh TH, Cao D, Shan QY, Bacon A, Hsu LY, Ooi EE. Acquired carbapenemases in enterobactericeae in Singapore, 1996-2012. Pathology. 2013;45(6):600-3. doi:10.1097/PAT.0b013e3283650b1e.

33. Gutiérrez-Gutiérrez B, Salamanca E, Pérez-Galera S, Bonomo R, Carmeli Y, Paterson $D$ et al. Assessment of betalactam/beta-lactamase inhibitor combinations for the treatment of bacteraemia due to extended-spectrum beta-lactamase-producing Enterobacteriaceae: The INCREMENT Project. Program and abstracts of the 54th Interscience Conference on Antimicrobial Agents and Chemotherapy (abstract K-1041). Washington, DC: American Society for Microbiology, 2014.

34. Deresinski S. In the literature. Piperacillin-tazobactam and extended-spectrum beta-lactamase--producing Escherichia coli. Clin Infect Dis. 2013;57(10):iii-iv.

35. Tamma PD, Han JH, Rock C, Harris AD, Lautenbach E, Hsu AJ, et al. Carbapenem therapy is associated with improved survival compared with piperacillin-tazobactam for patients with extended-spectrum beta-lactamase bacteremia. Clinical infectious diseases : an official publication of the Infectious Diseases Society of America. 2015. doi:10.1093/cid/civ003.

\section{Submit your next manuscript to BioMed Central and take full advantage of:}

- Convenient online submission

- Thorough peer review

- No space constraints or color figure charges

- Immediate publication on acceptance

- Inclusion in PubMed, CAS, Scopus and Google Scholar

- Research which is freely available for redistribution 